\title{
Yangian-invariant field theory of matrix-vector models
}

\author{
J. Avan * A. Jevicki ${ }^{\dagger} \quad$ J. Lee ${ }^{\ddagger}$
}

June 1996

\begin{abstract}
We extend our study of the field-theoretic description of matrix-vector models and the associated many-body problems of one dimensional particles with spin. We construct their Yangian-su $(R)$ invariant Hamiltonian. It describes an interacting theory of a $c=1$ collective boson and a $k=1 s u(R)$ current algebra. When $R \geq 3$ cubic-current terms arise. Their coupling is determined by the requirement of the Yangian symmetry. The Hamiltonian can be consistently reduced to finite-dimensional subspaces of states, enabling an explicit computation of the spectrum which we illustrate in the simplest case.
\end{abstract}

PACS Numbers: 03.65.Fd; 05.30.Fk; 11.25.Hf; 11.25.Sq; 11.30.Na.

Keywords: Collective theory; Matrix models; Calogero Moser model; Yangian invariance.

PAR LPTHE 96-22

${ }^{*}$ L.P.T.H.E. Université Paris VI (CNRS UA 280), Box 126, Tour 16, $1^{\text {er }}$ étage, 4 place Jussieu, 75252 Paris Cedex 05, France

${ }^{\dagger}$ Brown University Physics Department, Box 1843, Providence RI 02912-1843, USA

${ }^{\ddagger}$ Center for Theoretical Physics, Seoul National University, Seoul 151-742, KOREA 


\section{Introduction}

This work represents a continuation of a study started in [四] which dealt with a field-theoretic representation of coupled matrix-vector models and their associated many-body problems (Haldane-Shastry [2] and Calogero-Moser [3] models). The general structure, as emerged from [1] is characterized by an interacting theory of a collective boson with a set of variables realizing a current algebra. The bosonic field describes the dynamics of the eigenvalues of the original matrix field $M_{i j}$ and the current algebra is constructed from the matrix and quarklike degrees of freedom $\Psi_{a}(i)$. In a QCD-type terminology this represents an interaction of closed and open strings, the latter carrying $s u(R)$ Chan-Paton factors.

When the flavor degrees of freedom are further restricted to one fermion per site one obtains the spin Calogero-Moser model [4] in its particle-exchange version [5, 6, 7] which represents a system of $\mathrm{N}$ interacting particles with coordinates $\lambda_{i}$ and spins $\sigma_{i}$.

These theories have received major attention recently due to their exact classical and quantum solvability and the presence of exact Yangian symmetries [8, 9]. In the nondynamical (frozen eigenvalue) case the continuum Hamiltonian and Yangian structure written in terms of $k=1$ level Kac-Moody algebra was presented 10, 11, 12 and connected to the discrete dynamical models [2, 11]. Some aspects of the spectrum were investigated and described in terms of spinon vertex operators of the Kac Moody algebra [13, 6, 11].

In this work we discuss the full collective quantum field theory for the dynamical su $(R)$ spin theory. The core assumption for our construction of this theory will be the existence of an exact $s l(R)$ Yangian symmetry. In the simplest case of $s u(2)$ spins the structure given in [1] is then essentially complete. On the other hand it was shown in [15, 16] that in the case of $R \geq 3$ non-trivial quantum effects are present in the non-dynamical case. They require introducing higher-order terms in the Hamiltonian and the Yangian generators, representing zero modes of the higher-spin generators of $W_{R}$ algebras [17] constructed from the original currents by a standard procedure. The precise couplings can be determined through implementation of the Yangian symmetry [15] or through consistency of the effective spinon dynamics as a spin Calogero Moser system [16]. We establish a very similar structure in the full dynamical case. The Hamiltonian is represented in terms of of a $u(R)$ Kac-Moody algebra with the higher-order terms dictated by the requirement of Yangian symmetry. Since the variables of the Kac-Moody algebra are bosonic this represents a bosonization of the original fermionic system. t

The content of our paper is as follows. In Section 2 we recall the connection between the matrix-vector model and the spin Calogero-Moser model, restricted to the Haldane-Shastry exchange operator formulation. We then use the structure already deduced in [1] and the known results for the pure spin collective representation [10, including the cubic corrections [15, 16] for $R \geq 3$ to get a candidate Hamiltonian for the dynamical-eigenvalue problem.

In Section 3 we implement Serre's relation for the Yangian generators [19] with correction terms, and we determine the exact couplings of the Hamiltonian which guarantee commutation with these generators. This leads to a one-parameter family of Yangian-invariant models.

Section 4 and 5 are devoted to establishing the spectrum of this hamiltonian. We prove in Section 4 that the problem can be reduced to the diagonalization of finite-size matrices and we illustrate this in Section 5 by computing exactly the lowest-lying eigenvalues in the spinon formalism for one-spinon states. Consistency requirements on the rational nature of

\footnotetext{
${ }^{1} \mathrm{~A}$ possibly related approach can be found in 18
} 
the eigenvalues can then be used to further specify the values of the coupling coefficients. This specification will not be pursued to the end here, leaving this technical matter for further studies.

\section{From matrix models to field theory of spin Calogero- Moser models}

Let us first recall in more detail the way in which the spin Calogero-Moser model emerges from a coupled matrix-vector theory.

One starts from the matrix model Hamiltonian:

$$
\mathcal{H}=\operatorname{Tr} \frac{1}{2}\left(U^{-1} \frac{d U}{d t}\right)^{2}+V(U)+\sum_{a=1}^{\mathcal{N}} \Psi_{\alpha}^{\dagger} U \Psi_{\alpha}
$$

where $U(t)=\exp (i M(t)) ; M_{i j}(t), i, j=1 \cdots N$ is a hermitian $U(N)$ matrix and $\Psi_{\alpha}(i), \alpha=$ $1 \cdots R$ are the complex quark-like $U(N)$-vector fermionic degrees of freedom, independent of and commuting with $U$. The latter, in addition to their $U(N)$ vector index, also carry a flavor index $\alpha=1 \cdots R$.

The space of states is then consistently constrained by a $U(N)$ colour-singlet requirement expressed by Gauss's law (quantization of the momentum map) :

$$
(i[U, \tilde{P}]+\mathcal{Q})_{i j} \mid \text { state }>=0
$$

where $\mathcal{Q}_{i j} \equiv \sum_{a=1}^{R} \Psi_{a}^{\dagger}(i) \Psi_{a}(j)$ is the $U(N)$ color generator acting on quarks and $\tilde{P} \equiv$ $U^{-1} \dot{U} U^{-1}$ is the conjugate field to $U$. This singlet requirement is equivalent to a description of the restricted quantum system in terms of invariant quantities, leading to the corresponding many-body picture. Setting $M \equiv V \Lambda V^{\dagger} ; U \equiv V e^{i \Lambda} V^{\dagger}$ where $\Lambda$ is the diagonal matrix of eigenvalues $\lambda_{i}$ of $M$ and $V$ is a unitary matrix; $\Psi \equiv V \Psi^{\prime}$, Gauss' law is solved by:

$$
\tilde{P} \equiv V\left(\tilde{p}_{i} \delta_{i j}+i \frac{\mathcal{Q}_{i j}}{\omega_{i}-\omega_{j}}\right) V^{\dagger}
$$

where $\omega_{i}=e^{i \lambda_{i}}$ and $\tilde{p}_{i}$ are conjugate momenta of the eigenvalues $\omega_{i}$.

We drop from now on the potential terms in the Hamiltonian which contribute as external potentials/fields to the overall collective dynamics. The Hamiltonian $\operatorname{Tr}\left(\operatorname{Pe} e^{i M}\right)^{2}$ takes the form:

$$
H \equiv \frac{1}{2} \sum_{i=1}^{N} p_{i} \cdot p_{i}+\frac{1}{2} \sum_{i \neq j=1}^{N} \frac{\mathcal{Q}_{i j} \mathcal{Q}_{j i}}{\left(\omega_{i}-\omega_{j}\right)^{2}} \omega_{i} \omega_{j}
$$

where $p_{i}=\tilde{p}_{i} e^{i \lambda_{i}}$ is the conjugate momentum to $\lambda_{i}$. It now exhibits the trigonometric Calogero potential interaction in terms of the variables $\lambda_{i}$. To obtain the standard exchangeoperator formulation of the spin-spin interaction [6] one uses the fermion operator representation of the spin operators $\mathcal{Q}_{i j} \equiv \sum_{\alpha=1}^{R} \Psi_{\alpha}^{\dagger}(i) \Psi_{\alpha}(j)$ and one fixes to the same constant number the value of the number operator at each site. This "equal weight" requirement, which also occurred in [9] when computing commuting quantum Calogero-Moser Hamiltonians using the quantum $R$ matrix technique, reads: 


$$
\mathcal{N}_{i} \equiv \sum_{\alpha=1}^{R} \Psi_{\alpha}^{\dagger}(i) \Psi_{\alpha}(i)=1
$$

This requirement is consistent since $\mathcal{N}_{i}$ commutes with $H$ due to the $\mathcal{Q}_{i j} \mathcal{Q}_{j i}$ form of the interaction. This is equivalent to requiring that the trace (or $u(1)$ part) of the "flavour" $u(R)$ algebra, represented as $\mathcal{Q}_{\alpha \beta}(i) \equiv \Psi_{\alpha}^{\dagger}(i) \Psi_{\beta}(i)$ be frozen at each site. In terms of these local flavour generators the interaction part of the Hamiltonian reads:

$$
H_{\text {int }} \equiv \frac{1}{2} \sum_{i \neq j=1}^{N} \frac{\sum_{\alpha, \beta=1}^{R}\left(1-\mathcal{Q}_{\alpha \beta}(i) \mathcal{Q}_{\beta \alpha}(j)\right)}{\left(\omega_{i}-\omega_{j}\right)^{2}} \omega_{i} \omega_{j}
$$

and then becomes, using a well-known identity valid when the fermion number is 1 at each site :

$$
H_{\text {int }} \equiv \frac{1}{2} \sum_{i \neq j=1}^{N} \frac{\left(1-P_{i j}\right) \omega_{i} \omega_{j}}{\left(\omega_{i}-\omega_{j}\right)^{2}}
$$

Here the operator $P_{i j}$ permutes the spins of the $i$-th and $j$-th particle, while the wavefunctions are antisymetric under simultaneous permutation of coordinates and spins.

In terms of the standard notation [6, 7] this corresponds to the (fermionic) spin Calogero Moser model at coupling $\lambda=-1$.

The transition to a field-theoretical description is achieved through the introduction of two collective fields. The first one is built out of the density of eigenvalues:

$$
\Phi_{n} \equiv \operatorname{Tr}\left(U^{n}\right)=\sum_{i=1}^{N} \omega_{i}^{n}, n>0
$$

and its canonical conjugate $\Pi_{n}$. One introduces the decoupled combinations $\alpha_{n}\left(\tilde{\alpha_{n}}\right) \equiv \Phi_{n}-$ $(+) n \Pi_{n}$ for $n>0$ and their conjugate $\alpha_{n}\left(\tilde{\alpha_{n}}\right), n<0$ with resulting canonical commutation relations:

$$
\left[\alpha_{n}, \alpha_{m}\right]=n \delta_{n+m, 0}
$$

The commutator for the other chirality $\tilde{\alpha_{n}}$ has the opposite sign: $\left[\tilde{\alpha_{n}}, \tilde{\alpha_{m}}\right]=-n \delta_{n+m, 0}$. The other collective field is a set of currents built out of the $u(R)$ generators at each site:

$$
\mathcal{J}_{n}^{\alpha \beta} \equiv \sum_{i=1}^{N} \mathcal{Q}_{i}^{\alpha \beta} \omega_{i}^{n}
$$

Again one introduces two decoupled sets of fields $J, \tilde{J}$ realizing two Kac-Moody algebras with opposite central charge, such that $\mathcal{J}=J+\tilde{J}$. As presented in [1] the collective Hamiltonian for the matrix-vector model is then obtained by applying the operators on the reduced Hilbert space where it takes the form:

$$
H=\frac{1}{6} \sum_{n_{i} \in \mathbb{Z}} \alpha_{n_{1}} \alpha_{n_{2}} \alpha_{n_{3}} \delta_{n_{1}+n_{2}+n_{3}, 0}+\sum_{n \in \mathbb{Z}} \alpha_{n} T_{-n}(J)+\sum_{n \in \mathbb{N}^{*}} n J_{-n}^{a} J_{n}^{a}
$$

For simplicity we shall only study the Hamiltonian (11) which acts on states of fixed chirality for both $\Phi_{n}$ and $\mathcal{J}_{n}$ fields. We shall later discuss the full Hamiltonian and establish the consistency of this requirement. The first and last term of this Hamiltonian are standard 
terms from collective theories of pure matrix models [20] and pure spin models 10 . The Roman indices $a$ characterize here and from now on the adjoint representation of $u(R)$. We now take into account the constraint (one fermion per site) which is to be imposed to get the Haldane-Shastry formulation of the spin-spin interaction. In terms of the current this constraint reads:

$$
J_{n}=\sum_{\alpha=1}^{R} J_{n}^{\alpha \alpha}=\sum_{i=1}^{N} 1 . \omega_{i}^{n}=\phi_{n}, n>0 .
$$

This implies that the $c=1$ scalar boson is to be identified with the $U(1)$ piece of the current algebra. What remains is now a $s u(R)$ current algebra, which according to [10] is to be taken at level $k=1$ :

$$
\left[J_{n}^{a}, J_{m}^{b}\right]=f_{c}^{a b} J_{n+m}^{c}+n \delta_{n+m, 0} .
$$

The Hamiltonian structure (11) is the result of ref [1]. We now recall that for $R \geq 3$ higher order (cubic) counterterms are required already in the pure spin case 15, in the definition of the Hamiltonian and the Yangian generators. In our approach such terms can be interpreted as coming from the non-linear Jacobian which typically appears in any collective field formulation owing to the non-trivial change of variables [20]. Rather than proceeding to derive the Jacobian explicitely it is simpler to follow the strategy of [15] and specify the additionnal terms from the requirement of Yangian symmetry. We shall soon see that this approach allows for a supplementary parameter in the Hamiltonian, leading to the general form:

$$
\begin{aligned}
H & =\frac{b}{6} \sum \alpha_{n_{1}} \alpha_{n_{2}} \alpha_{n_{3}} \delta_{n_{1}+n_{2}+n_{3}, 0}+\sum_{n \in \mathbb{Z}} b \alpha_{n} T_{-n}(J)+\sum_{n \in \mathbb{N}^{*}} n \alpha_{-n} \alpha_{n} \\
& +\sum_{n>0} n J_{-n}^{a} J_{n}^{a}+\frac{\gamma R}{(R+1)(2+R)} W_{0}
\end{aligned}
$$

where $\gamma^{2}=1+\frac{b^{2}}{R}$. One also expects that the Hamiltonian associated to the spin Calogero Moser model belongs to this family. We shall discuss this point later.

We have here explicitely separated the $U(1)$ piece of the current algebra as $\sum_{n>0} n \alpha_{-n} \alpha_{n}$ and we have introduced the energy-momentum tensor $T^{J}(z)$ and spin-3 $W$-algebra generator built out of the current algebra $s u(R)$ :

$$
\begin{aligned}
T_{n}^{J} & =\frac{1}{2(N+1)} \sum_{a, b} d^{a b}\left(J^{a} J^{b}\right)_{n} \\
W_{0} & =\frac{1}{6} d^{a b c}\left(J^{a}\left(J^{b} J^{c}\right)\right)_{0}
\end{aligned}
$$

The tensor $d^{a b}$ is of course the Killing form and $d^{a b c}$ is the invariant symmetric three-form defined in Appendix B. From now on the brackets () enclosing products of two or three $J^{a}$ fields will indicate the normal ordered products defined in Appendix A.

We shall later denote by $H_{\alpha}$ the pure scalar Hamiltonian; by $H_{J}$ the pure spin Hamiltonian; and by $H_{\text {int }}$ the interaction $\alpha J$ Hamiltonian in (14).

Introducing an explicit conformal field notation as : 


$$
\begin{gathered}
\alpha(z)=\sum_{n \in \mathbb{Z}} z^{-n-1} \alpha_{n} \quad ; \quad J^{a}(z)=\sum_{n \in \mathbb{Z}} z^{-n-1} J_{n}^{a} \\
T^{J}(z)=\frac{1}{2(1+R)}\left(J^{a} J^{b} d^{a b}\right)(z) \quad ; \quad W(z)=\frac{1}{6} d^{a b c}\left(J^{a}\left(J^{b} J^{c}\right)\right)(z)
\end{gathered}
$$

one rewrites the Hamiltonian as the integral of a local and bilocal field density:

$$
\begin{aligned}
H & =\int d z b\left(z^{2} \frac{1}{6}(\alpha(z))^{3}+\alpha(z) T^{J}(z)\right)+\gamma \frac{R}{(R+1)(R+2)} W(z) \\
& +\int d z \int d w\left(\frac{d^{a d} J^{a}(w) J^{d}(z)}{(w-z)^{2}}+\frac{\alpha(z) \alpha(w)}{(w-z)^{2}}\right)
\end{aligned}
$$

As indicated above there exists two chiralities for the bosonic scalar fields $\alpha, \tilde{\alpha}$. The Hamiltonian (17) corresponds to the choice of $\alpha$ with $\left[\alpha_{n}, \alpha_{m}\right]=n \delta_{n+m, 0}$. The opposite chirality boson obeys the opposite-sign commutation relation $\left[\tilde{\alpha}_{n}, \tilde{\alpha}_{m}\right]=-n \delta_{n+m, 0}$. When coupled to the current algebra (13) with central charge $k=1$ the resulting Hamiltonian looks very similar to the one in eq.(14) although with modified signs for the second and third term: $-\sum \tilde{\alpha}_{n} T_{-n}(J)-\sum_{n>0} n \tilde{\alpha}_{-n} \tilde{\alpha_{n}}$, and a modified coupling $\tilde{\gamma}= \pm \sqrt{1-\frac{b^{2}}{R}}$. Finally if one chooses the current algebra $J_{n}^{a}$ with negative central charge $k=-1$ one gets the same Hamiltonian up to an overall sign.

The full non-relativistic Hamiltonian is ultimately to contain two Hamiltonians of the type (14) describing the physics of the matrix-vector model in the continuum fermion representation near the upper and lower Fermi surface respectively [21]. We recall that the non-relativistic collective density reads $\Phi=\alpha+\tilde{\alpha}$ and the non-relativistic current density reads $\mathcal{J}^{a}=J^{a}+\tilde{J}^{a}$ where $J$ obeys the $k=1$ current algebra (13) and $\tilde{J}$ obeys the $k=-1$ current algebra. The full Hamiltonian is then expected to read:

$$
H(\phi, \mathcal{J})=H(\alpha, J)+H(\tilde{\alpha}, \tilde{J})
$$

with the coupling coefficients $b$ and $\gamma, \tilde{\gamma}$ determined in our previous discussion. This separation of chiralities is a known feature of collective field theory. Indeed the spinless part of (18) yields the separated, pure one-matrix collective Hamiltonian [20]:

$$
H=\int \frac{1}{6}\left(\alpha^{3}(z)-\tilde{\alpha}^{3}(z)\right) d z
$$

It is this general feature which allows a consistent restriction of our study (spectrum, symmetry algebras) to one chirality.

\section{$3 \quad$ Yangian symmetry}

\subsection{The Yangian generators}

In this section we fix the central charge of the Kac-Moody algebra $s u \widehat{(R})_{k}$ to be $k=1$. For purposes of generalization the central charge of the scalar field $\alpha$ shall be taken to be $q \in \mathbb{R}$ and $\left[\alpha_{n}, \alpha_{m}\right]=n q \delta_{n+m, 0}$. We shall see that in fact only the sign of $q$ is a relevant quantity and $q$ can be taken as \pm 1 . 
The presence of a large symmetry algebra in the theory is most easily seen in the matrix description [9, 22]. At the classical level the $U(N)$ invariant generators $Q_{n}^{\alpha, \beta} \equiv \Psi_{\alpha}^{\dagger}\left(P e^{i M}\right)^{n} \Psi_{\beta}$ Poisson-commute with the Hamiltonian due to the $r$-matrix structure [9], and realize a (quadratic) $\operatorname{sl}(R)$ Yangian algebra denoted $\mathcal{Y}(\operatorname{sl}(R))$ [19:

$$
\begin{aligned}
\left\{Q_{n}^{\alpha \beta}, Q_{m}^{\gamma \delta}\right\} & =\sum_{i=1}^{n} Q_{n+m-i}^{\gamma \beta} Q_{i-1}^{\alpha \delta}+Q_{n-i}^{\gamma \beta} Q_{m+i-1}^{\alpha \delta} \\
& +\sum_{j=1}^{m} Q_{n+j-1}^{\gamma \beta} Q_{m-j}^{\alpha \delta}+Q_{j-1}^{\gamma \beta} Q_{m+n-j}^{\alpha \delta}
\end{aligned}
$$

At the quantum level [8, 10, 7] similar formulae hold for the first and second Yangian generator:

$$
\begin{aligned}
Q_{0}^{\alpha \beta} & =\Psi_{\alpha}^{\dagger} \Psi_{\beta} \\
Q_{1}^{\alpha \beta}=\sum_{i=1}^{N} p_{i} E_{i i}^{\alpha \beta} & -\frac{1}{2} \sum_{k \neq j=1}^{N}\left(E_{j j} E_{k k}\right)^{\alpha \beta} \frac{e^{2 \lambda_{i}}+e^{2 \lambda_{j}}}{e^{2 \lambda_{i}}-e^{2 \lambda_{j}}}
\end{aligned}
$$

where as usual $E_{i j}^{\alpha \beta} \equiv \Psi_{i}^{\alpha \dagger} \Psi_{j}^{\beta}$.

It was also established [16, 15 that the pure-spin continuous system had a classical and quantum symmetry algebra $\mathcal{Y}(\operatorname{sl}(R))$ generated by the zero mode $Q_{0}^{a}$ of the current field $J^{a}(z)$, and the modified generator (for $R>2$ ):

$$
\tilde{Q}_{1}^{a}=f^{a b c} \sum_{n \geq 0} J_{-n}^{b} J_{n}^{c}-\frac{R}{R+2} d^{a b c}\left(J^{b} J^{c}\right)_{0}
$$

Here $f^{a b c}$ are the structure coefficients of the Lie algebra $s l(R) ; d^{a b c}$ is the symmetric 3tensor defined in Appendix B and the bracket notation, as in the Hamiltonian (14), denotes the normal-ordering defined in Appendix A. As before, Roman indices $a, b, c$ characterize the adjoint representation of $s u(R)$.

From the original definition of (20) as the Hamiltonian-reduced version of the generator $\operatorname{Tr}\left(\Psi^{\alpha \dagger} P e^{i M} \Psi^{\beta}\right)$ we are lead to a continuum limit for the non-trivial Yangian generator $Q_{1}^{a}$ with a similar supplementary term of the form $d(J J)_{0}$. :

$$
Q_{1}^{a}=b \sum_{n \in \mathbb{Z}} \alpha_{-n} J_{n}^{a}+f^{a b c} \sum_{n>0} J_{-n}^{b} J_{n}^{c} \pm \nu\left(\frac{R}{R+2}\right) d^{a b c}\left(J^{b} J^{c}\right)_{0}
$$

To simplify further notations we define the field $W^{a} \equiv d^{a b c}\left(J^{b} J^{c}\right)$. We have introduced here two arbitrary normalizations $b$ and $\nu$ in order to consider the most general ansatz combining the three monomial operators. We now prove the first major result:

Proposition 1: The operators $\left\{Q_{0}^{a}=J_{0}^{a}, Q_{1}^{a}\right\}$ generate the Yangian algebra $\mathcal{Y}(\operatorname{sl}(R))$ iff $\nu^{2}=1+\frac{b^{2} q}{R}$.

The proof follows on similar lines as in [15] and relies heavily on a number of identities mentioned in that reference and recalled in Appendix B, C and D.

We need to check Serre's relations for these generators. For $R \geq 3$ only three relations are independent. The first two read: 


$$
\begin{aligned}
& (S 1)\left[Q_{0}^{a}, Q_{0}^{b}\right]=f^{a b c} Q_{0}^{c} \\
& (S 2)\left[Q_{0}^{a}, Q_{1}^{b}\right]=f^{a b c} Q_{1}^{c}
\end{aligned}
$$

They are obvious consequences of the definitions of $Q_{0}^{a}, Q_{1}^{b}$. The third relation is

(S3) $\left[Q_{1}^{a},\left[Q_{1}^{b}, Q_{0}^{c}\right]\right]+$ cyclic permutations on a, b, c $=\mathrm{A}^{\text {abc,def }}\left\{\left\{\mathrm{Q}_{0}^{\mathrm{a}}, \mathrm{Q}_{0}^{\mathrm{b}}, \mathrm{Q}_{0}^{\mathrm{c}}\right\}\right\}$

where $A^{a b c, d e f}$ is a 6-index tensor defined as $\sum_{p, q, r} f^{a d p} f^{b c q} f^{c f r} f^{p q r}$ and $\{\{\}\}$ denotes the totally symmetrized product.

The proof of $S 3$ follows from two basic steps:

Step 1: Compute the contracted commutator $f^{d b c}\left[Q_{1}^{a}, Q_{1}^{d}\right]$ and substract contributions from the exact pure-spin operator defined in [15] which gives the exact r.h.s. of $S 3$.

Step 2: Show that the remaining contributions vanish.

Both steps are greatly simplified by the property: S3.

(P1) Any contribution $f^{a d X}$ from the commutator $\left[Q_{1}^{a}, Q_{1}^{b}\right]$ vanishes from the l.h.s. of

This property is an easy consequence of the Jacobi identity (see Appendix B, id.J1).

Step 1 is achieved as follows. The contribution to $S 3$ of the original two operators in (21) is obtained from the commutators $C 5, C 6$ in Appendix C:

$$
\left.\nu^{2}\left[W^{a}, W^{d}\right]+(\text { cross }- \text { terms }=0)+(\text { full commutator [fJJ }, \mathrm{fJJ}]\right)
$$

The full commutator $[f J J, f J J]$ computed in Appendix $C 4$ gives the correct r.h.s. contribution to Serre's relation plus an extra piece, partially cancelled (when $\nu^{2} \neq 1$ ) up to a null field, by the $[W, W]$ term. This leaves an unaccounted-for contribution:

$$
S_{3}^{(1)}=\left(1-\nu^{2}\right) \frac{1}{6}\left(f^{a b c} f^{c e g} f^{d e f}-(a \leftrightarrow d)\right)\left(J^{b}\left(J^{f} J^{g}\right)\right)_{0}
$$

The extra term $\alpha J$ in (22) contributes as:

$$
S_{3}^{(2)}=b^{2} q \sum_{m} m J_{m}^{a} J_{-m}^{d}+f^{a d X}-\text { type contributions }
$$

Indeed the term $\left[b \sum \alpha_{-n} J_{n}^{d}, b \sum \alpha_{-m} J_{m}^{a}\right]$ yields immediately the non-trivial contribution $b^{2} q \sum_{m} m J_{m}^{a} J_{-m}^{d}$ (from $\alpha$ commutators) plus an $f^{a d X}$ term. The other contribution:

$$
\left[b \sum \alpha_{-n} J_{n}^{d}, \sum_{m>0} f^{a b c} J_{-m}^{b} J_{m}^{c}\right]-(a \leftrightarrow d)
$$

is computed as:

1) an $n=0$ contribution $\alpha_{0}\left[J_{n}^{d}, \sum_{m>0} f^{a b c} J_{-m}^{b} J_{m}^{c}\right]=\alpha_{0} f^{a d e} Q_{1}^{e}$ through $S 2$, giving again $f^{a d X}$.

2 ) the $n>0$ and $n<0$ contribution. Reorganizing indices leads to two terms:

$\alpha_{-n}\left(\sum_{m>0}\left(f^{a b c} f^{d b e}+f^{a e b} f^{d b c}\right) J_{-m}^{e} J_{m+n}^{c}\right)$, which by Jacobi identity $J 1$ (App. B) gives $f^{a d b} f^{e b c} \cdots$ 
$\alpha_{-n}\left(\sum_{n \geq m>0}\left(f^{a b c} f^{d b e}-(a \leftrightarrow d)\right) J_{n-m}^{e} J_{m}^{c}\right)$ which can be reexpressed as a commutator by a suitable change of indices $c \leftrightarrow e$ :

$$
\alpha_{-n}\left(\sum_{n \geq m>0}\left(f^{a b c} f^{d b e}\left[J_{n+m}^{e}, J_{m}^{c}\right]\right)\right.
$$

Through the normalization identity N4 (App. B) this is proportional to $f^{a d m}$. This ends Step 1.

We have given here a rather detailed account of one of the many computations included in our derivation. We shall not be so detailed in the next proofs and shall only indicate the relevant normalizations, Jacobi identities or null field identities relevant for our purposes.

Step 2 now consists in computing exactly $S_{3}^{(1)}$. This is essentially achieved by using $J 3$ (Appendix B) to reexpress products of two $f^{x y z}$ structure constants with one common index, here $f^{c E g} f^{d E f}$. The $\delta$ term in $J 3$ leads to:

$$
-4\left(1-\nu^{2}\right) \frac{1}{6}\left(\partial J^{a} J^{d}-\partial J^{d} J^{a}\right)_{0}
$$

The $d$ terms require a very intricate and cumbersome treatment leading ultimately to:

$$
4\left(1-\nu^{2}\right)\left(\frac{R}{8}+\frac{1}{6}\right)\left(\partial J^{a} J^{d}-\partial J^{d} J^{a}\right)_{0}+\frac{1}{6} \Phi_{3}
$$

where $\Phi_{3}$ is the null field defined in $F 2$ (Appendix C).

If one now assumes that $1-\nu^{2}=-\frac{b^{2} q}{R}$ one obtains for $S_{3}^{(1)}$ the expression $\frac{b^{2} q}{2}\left(\partial J^{a} J^{d}-\right.$ $\left.\partial J^{d} J^{a}\right)_{0}$ which from the definition of normal order $A 1$ (App. A) and the conformal dimension $\Delta(\partial J)=2$ is rewritten as:

$$
-\frac{b^{2} q}{2}\left\{\sum_{n<0}\left(-2 n J_{n}^{a} J_{-n}^{d}-2 n J_{-n}^{d} J_{n}^{a}\right)+\left[J_{0}^{a}, J_{0}^{d}\right]\right\}
$$

and finally, extracting the non-relevant contribution $f^{a d X}$ from the $\left[J_{0}^{a}, J_{0}^{d}\right]$ term and the reordering of $J_{-n}^{d} J_{n}^{a}$ to $J_{n}^{a} J_{-n}^{d}$, one obtains $-b^{2} q \sum_{n>0, n<0} n J_{n}^{a} J_{-n}^{d}$, thereby cancelling $S_{3}^{(2)}$.

We have thus proved that Serre's relations were obeyed by the generators (20) iff $\nu^{2}=$ $1+\frac{b^{2} q}{R}$. We immediately note that $|q|$ can be eliminated from (22) by redefining $\alpha\left(U(1)_{q}\right)=$ $\sqrt{|q|} \alpha\left(U(1)_{ \pm 1}\right)$ and $b^{\prime}=b \sqrt{|q|}$.

\subsection{The Hamiltonian}

We now discuss the Yangian invariance of the Hamiltonian. As indicated in Section 2, the quantum non-hermitian Hamiltonian, directly derived from the finite discrete case by replacement of the various terms with their assumed continuum equivalents, is not Yangianinvariant when $R \geq 3$. It requires in the pure spin case an additional term, cubic in the currents and proportionnal to the spin-3 generator of the $W_{R}$ algebra naturally associated to the $\operatorname{sl}(R)$ current algebra $J^{a}(z)$. In the dynamical case we were lead to the form (14) which we now supplement by adding arbitrary normalization coefficients in front of all monomials but one: 


$$
\begin{aligned}
H & =\frac{\lambda_{1}}{6} \sum \alpha_{n_{1}} \alpha_{n_{2}} \alpha_{n_{3}} \delta_{n_{1}+n_{2}+n_{3}, 0}+\lambda_{2} \sum_{n} \alpha_{n} T_{-n}(J)+\sum_{n>0} \lambda_{3} n \alpha_{-n} \alpha_{n} \\
& +\sum_{n>0} n J_{-n}^{a} J_{n}^{a}+\frac{\gamma R}{(R+1)(R+2)} W_{0}
\end{aligned}
$$

We now prove the second essential result of this paper:

Proposition 2: $\left[H, Q_{1}^{a}\right]=0$ iff $\lambda_{1}=\frac{b}{q} ; \lambda_{2}=b ; \lambda_{3}=\frac{1}{q} ; \gamma=\nu$ and $\gamma^{2}=1+\frac{b^{2} q}{R}$. Since $H$ is obviously $s l(R)$-invariant this proves its Yangian invariance.

The proof of Proposition 2 follows similar lines as Proposition 1. The first step consists in evaluating the commutator and substracting the pure-spin contributions already known [15] to cancel up to a null field $\Xi(x)$ (see $F 1$, Appendix C). The full commutator is a lengthy expression but it is immediately seen that: cross-terms in $\alpha^{2} J$ vanish iff $\lambda_{1} q=\lambda_{2}$; crossterms $\alpha J J$ vanish iff $\lambda_{2}=b$; cross-terms $\alpha J$ vanish iff $\lambda_{3}=\frac{1}{q}$; cross-terms $\alpha W^{a}$ vanish iff $\gamma=\nu$. The remaining terms are then easily recast as:

$$
-\sum_{n \in \mathbb{Z}} q b^{2} n J_{n}^{a} T_{-n}^{J}\left(\text { from }\left[\alpha \mathrm{T}^{\mathrm{J}}, \alpha \mathrm{J}^{\mathrm{a}}\right]\right)-\left(1-\gamma^{2}\right) \frac{1}{3} \mathrm{f}^{\mathrm{abc}} \mathrm{f}^{\mathrm{cde}}\left(\partial \mathrm{J}^{\mathrm{b}}\left(\mathrm{J}^{\mathrm{d}} \mathrm{J}^{\mathrm{e}}\right)-\mathrm{J}^{\mathrm{b}}\left(\partial \mathrm{J}^{\mathrm{d}} \mathrm{J}^{\mathrm{e}}\right)\right)_{0}
$$

The second term in (34) results from the incomplete cancellation (up to the null field $\Xi$ ) of the full commutator $[f J J, J J]$ with the renormalized contribution $\gamma^{2}\left[W_{0}, W^{a}\right]$.

The second step of the proof now consists in reevaluating the $f f$ term in (34), essentially using the Jacobi product formula $J 3$ in Appendix B. The $\delta$ terms in $J 3$ contribute as:

$$
\frac{2}{R}\left\{\left(\partial J^{a}\left(J^{b} J^{b}\right)+\left(J^{a}\left(\partial J^{b} J^{b}\right)\right)\right\}=\frac{4(1+R)}{R} \sum_{n \in \mathbb{Z}}-n\left(J_{n}^{a} T_{-n}^{J}\right)\right.
$$

The $d^{x y z}$ terms contribute as :

$$
-\frac{(R+2)^{2}}{R^{2}}\left(d^{a b c} \frac{R^{2}}{2(R+2)^{2}}\left(-\frac{2}{3}\left(\partial J^{c} W^{b}+\partial W^{b} J^{c}\right)+\frac{1}{3}\left(\partial W^{b} J^{c}+J^{c} \partial W^{b}\right)\right)+\frac{R(R+2)}{12} \partial^{3} J^{a}\right)
$$

reproducing exactly the last terms in the null field $\Xi$. It follows that the $f f$ terms can be rewritten in a rather spectacular form as:

$$
-\frac{1}{3 R} f^{a b c} f^{c d e}\left(\partial J^{b}\left(J^{d} J^{e}\right)-J^{b}\left(\partial J^{d} J^{e}\right)\right)_{0}=\sum_{n \in \mathbb{Z}} n J_{n}^{a} T_{-n}^{J}-\left.\frac{(R+2)^{2}}{4 R(R+1)} \Xi(z)\right|_{0}
$$

All ( ) in these formulas denote the normal-ordered product recalled in Appendix A. Extensive use was made in these derivations of the normal-ordered commutators defined in $A 1$ and the OPE in $O 1-3$.

Plugging back (37) into (34) show immediately that the supplementary contributions to the commutator $\left[H, Q_{1}^{a}\right]$ also cancel up to the null field $\Xi$ in $F 4$, Appendix $\mathrm{C}$, iff the coupling $\gamma$ is such that $\gamma^{2}=1+\frac{b^{2} q}{N}$. This provides us with a non-trivial check on the consistency 
of our approach since both Serre's relations and subsequent Yangian invariance require the same constraint on the coupling $\gamma$. The generic Hamiltonian now takes the form:

$$
\begin{aligned}
H & =\frac{b}{6} \sum \alpha_{n_{1}} \alpha_{n_{2}} \alpha_{n_{3}} \delta_{n_{1}+n_{2}+n_{3}, 0}+b \sum_{n} \alpha_{n} T_{-n}^{J}+\sum_{n>0} n \alpha_{-n} \alpha_{n} \\
& +\sum_{n>0} n J_{-n}^{a} J_{n}^{a}+ \pm \sqrt{1+\frac{b^{2}}{R}} \frac{R}{(R+1)(R+2)} W_{0}
\end{aligned}
$$

As in the Yangian generator the parameter $|q|$ is irrelevant: it is eliminated by redefining $\alpha$ and $b$ in the same way. Only the choice $q= \pm 1$ is relevant. This particular Hamiltonian corresponds to the choice $q=1$.

The final result is the construction of a one-parameter family of Hamiltonians exhibiting an exact Yangian $\mathcal{Y}(s l(R)$ symmetry. The Hamiltonian takes the form of a non-zero-mode (bilocal) quadratic self-interaction of a $s u\left(\widehat{R}_{k=1} \times U(1)_{q=1}\right.$ current, supplemented by cubic terms $\alpha^{3}, \alpha(J J)$ and $J J J$ ensuring Yangian invariance, normalized by a three-dimensional one-parameter coupling $\left(b, 1, \pm \sqrt{1+\frac{b^{2}}{R}}\right)$. The Yangian generator $Q_{0}^{a}$ is the standard current generator; the non-trivial generator $Q_{1}^{a}$ is a sum of three quadratic terms $\alpha J, f J J$ and $d J J$ normalized by the same three-dimensional one-parameter coupling. In particular the sign \pm in front of $\sqrt{1+\frac{b^{2}}{R}}$ is to be the same.

When one chooses instead $q=-1$ (other chirality), the previous computations lead to

the relevant consistent value $\gamma= \pm \sqrt{1-\frac{b^{2}}{R}}$ together with changes of relative sign between the $\alpha^{3}$ term and the $\alpha T^{J}$ term, and between the $n \alpha \alpha$ term and the $n J J$ term. We shall not consider this case in our computations of the spectrum. It may be treated in an almost identical way and gives rise to a similar spectrum.

Before switching to the study of the spectrum of such theories it is interesting to note that one can also introduce Virasoro algebra generators involving both fields, as $T_{n}=\sum_{q} \alpha_{n-q} \alpha_{q}+$ $T_{n}^{J}$. The generator $T_{0}$ is easily seen to commute with both $H$ and $Q_{1}^{A}$ in their implemented version, thereby guaranteeing the conformal properties of the theory.

\section{Space of states and action of the Hamiltonian}

The spectrum of the Hamiltonian will be organized in multiplets of the Yangian. At this time we are mostly interested in finding explicit eigenstates and eigenvectors. The possibility of restricting the Hamiltonian to finite-dimensional vector spaces of states greatly facilitates such a construction.

\subsection{Reordering procedures}

The space of states on which we are going to apply (14) has an obvious tensor product structure. The first factor is the Fock space for the oscillator scalar operators $\alpha_{n}$. We define the vacuum $\mid 0>_{s c}$ to be annihilated by the positive modes of $\alpha(z)$. The zero-mode $\alpha_{0}$ is a $c$-number which we take to be 0 for simplicity. Negative modes build the Fock space and due to their canonical free-boson commutation relations, Wick theorem allows to write all states as linear combinations of ordered states: 


$$
\alpha_{-n_{p}} \cdots \alpha_{-n_{1}} \mid 0>_{s c} ; n_{p} \geq \cdots \geq n_{1}>0
$$

The second factor is the state space for the current algebra $\left\{J_{n}^{a}\right\}$. We choose the spinon representation of states since it is easy to handle and moreover should ultimately provide us with a way of comparing the results of the continuum dynamical theory with those of the continuum pure-spin theory expressed in this language [11, 16]. Spinon fields are intertwining chiral vertex operators [13, 11, 12, 16, 14]. The representation of the Lie algebra $s u(R)$ in which they live will be denoted by $\lambda$; the representations of the Kac-Moody algebra $s u(\widehat{R})_{k=1}$ which they connect will be denoted respectively $\sigma$ (object) and $\rho$ (image), according to the underlying representation of $s u(R)$. In the general case and when $k=1$, the spinon fields live in $R-1$ representations of $s u(\hat{R})_{k=1}$ aside from the vacuum representation [23]. They are labeled by the fundamental weights of $s u(R)$. A particular role is played by spinon fields, systematically used in [11, 14], which live in the contravariant vector $(\bar{R})$ representation of $s u(R)$. We shall come back to it at the end of this subsection. Note in this respect that, as pointed out in [14], the covariant representation $R$ is not on the same footing as the contravariant one owing to the Yangian symmetry which does not commute with conjugation.

The mode expansion of general spinon fields then reads:

$$
\Phi\left(\begin{array}{c}
\lambda \\
\rho \sigma
\end{array}\right)=\sum_{n \in \mathbb{Z}} \Phi\left(\begin{array}{c}
\lambda \\
\rho \sigma
\end{array}\right)-n-\Delta(\rho)+\Delta(\sigma) z^{n+\Delta(\rho)-\Delta(\sigma)-\Delta(\lambda)}
$$

where $\Delta($.$) denotes the conformal weights of the chosen representation \Delta(\lambda)=\frac{C(\lambda)}{2(R+1)}$, C being the second Casimir operator.

The vacuum $\mid 0>_{s p}$ is annihilated by positive-mode spinons. Multispinon states are obtained by concatenation of one-spinon modes with consistent fusion rules. In particular, given a state of the form $\Phi_{N_{k}}^{k} \cdots \Phi_{N_{1}}^{1} \mid 0>_{s p}$, consistency requires that $\sigma_{1}$ be the vacuum representation 1 and that $\rho_{q-1} \equiv \sigma_{q}$ for $q=1 \cdots k$. Other requirements will be mentioned later when required by the particular context.

One must beware that we are not constructing a free module but we must divide by the ideal generated by the commutation relations and (for $R \geq 3$ ) the null fields, as usual in a Verma module-type construction. Our resulting space of states is the sum of direct tensor products of the free boson module with all distinct irreducible pure current algebra modules constructed with spinon operators. On such a space of states the commutation relations leading to the Yangian invariance of the full Hamiltonian are therefore valid since they require only the use of the null field structure for the pure current algebra.

For our next purposes we need a more precise description of the commutation relations following from the OPE of spinon fields. They are characterized by the fact that they generically do not exhibit isolated integer-order poles but rather branch points and branch cuts of order $\frac{1}{R}$, characterizing the semionic nature of spinons [13]. As a consequence the commutation relations assume a quadratic-algebra form of the type:

$$
\Phi_{-M} \Phi_{-N}=\Phi_{-N} \Phi_{-M}+T_{M N} \delta_{M+N, 0}+\sum_{l \in \mathbb{N}^{*}} \Phi_{-M-l} \Phi_{-N+l}-\Phi_{-N-l} \Phi_{-M+l}
$$

We have not made explicit here the precise representation content of the spinon fields nor the exact form of the constant "commutator" tensor $T$ which in some cases may even vanish as we shall later see. The mode indices $M, N$ implicitely contain also the required shifts by conformal dimensions. However the essential feature of (41) for our future purpose, 
is the fact that only positive-l shifts arise in the r.h.s. This will now allow us to prove the central reordering lemma:

Proposition 3: Multispinon states can always be rewritten as linear combinations of fully mode-ordered "base states" of the form:

$$
\Phi_{-n_{p}}^{\Sigma_{p}} \cdots \Phi_{-n_{1}}^{\Sigma_{1}} \mid 0>_{s p}
$$

Here $\Sigma_{q}, q=1 \cdots p$ denotes the three representations parametrizing any spinon field; $n_{q}$ are positive integers such that $n_{p} \geq \cdots n_{1} \geq 0$ and dependance of the mode-index $n_{q}$ in the conformal weights has been omitted for simplicity (it may lead to slight modifications of the minimal value of the difference between the modes of two neighboring spinon operators).

Corollary 1: Since $\mid 0>_{s p}$ is annihilated by positive-mode spinons the modes in these "base states" are all negative up to a possible bounded shift by the conformal weight. Moreover multispinon states with a positive overall sum of modes are necessarily null states. Indeed reordering using (41) conserves the sum of modes. Hence the highest mode in such a reordered multispinon state must be positive and therefore annihilates the vacuum.

Proof of Proposition 3: By recursion on the number $N$ of one-spinon modes.

a) $N=1$ : one-spinon states are automatically ordered.

b) $N=N_{0}+1$ with ordering lemma and its corollary 1 assumed up to $N_{0}$. Consider now the state:

$$
\Phi_{-n_{N_{0}+1}} \Phi_{-n_{N_{0}}} \cdots \Phi_{-n_{1}} \mid 0>_{s p}
$$

with $n_{N_{0}} \geq \cdots \geq n_{1} \geq 0$

If $n_{N_{0}+1} \geq n_{N_{0}}$ the state (43) is ordered. If not we apply (41) to get:

$$
\begin{aligned}
\Phi_{-n_{N_{0}}} \Phi_{-n_{N_{0}+1}} \cdots \Phi_{-n_{1}} \mid 0>_{s p} & +T \cdots\left|0>_{s p}+\sum_{l>0} \Phi_{-l-n_{N_{0}+1}} \Phi_{l-n_{N_{0}}} \cdots \Phi_{-n_{1}}\right| 0>_{s p} \\
& +\sum_{l>0} \Phi_{-l-n_{N_{0}}} \Phi_{l-n_{N_{0}+1}} \cdots \Phi_{-n_{1}} \mid 0>_{s p}
\end{aligned}
$$

The second term in (44) can immediately be reordered as a $\left(N_{0}-1\right)$-spinon state. In the third and fourth terms, owing to Corollary 1 the sum of modes of the $N_{0}$ first terms cannot be allowed to become positive hence only the values of $l$ smaller then $\sum_{p=1}^{N_{0}} n_{p}$ may be taken into account. Similarly in the fourth term only values of $l$ smaller than $\sum_{p=1, p \neq N_{0}}^{N_{0}+1} n_{p}$ will contribute. Hence (44) is in fact a finite sum.

To the $N_{0}$ first spinon operators in all terms of (44) can now be applied the reordering lemma, leaving us with:

$$
\begin{aligned}
\Phi_{-n_{N_{0}}}[\text { ordered state }] \mid 0>_{\mathrm{sp}} & +\sum_{l>0}^{L_{0}} \Phi_{-l-n_{N_{0}+1}}[\text { ordered state }] \mid 0>_{\mathrm{sp}} \\
& +\sum_{l>0}^{L_{0}^{\prime}} \Phi_{-l-n_{N_{0}}}[\text { ordered state }] \mid 0>_{\mathrm{sp}}
\end{aligned}
$$


We now have a finite sum of $\left(N_{0}+1\right)$-spinon states. Each may be already fully ordered or not depending whether the last spinon mode is lower than its immediate predecessor. One then needs to reapply Procedure (43) $\rightarrow$ (46). But this reordering procedure which lead from (43) to (46) has in every case decreased strictly the value of the mode of the final spinon from $-n_{N_{0}+1}$ to either $-n_{N_{0}}$ or $-n_{N_{0}}-l$ or $-n_{N_{0}+1}-l$ with $l>0$.

On the other hand since the overall sum of modes is fixed, the last mode cannot decrease beyond the value $-\sum_{q=1}^{N_{0}+1} n_{q}$ otherwise the remaining $N_{0}$ spinon state would have an overall positive mode sum and thus would vanish following Corollary 1.

It follows that this procedure cannot be applied more than $\sum_{q=1}^{N_{0}+1} n_{q}$ times. Since it only stops when either the considered state is fully reordered or has become a null state, the final result, reached after a finite number of steps, is a finite sum of fully reordered $\left(N_{0}+1\right)-$ spinon states. This completes the recursion and proves the reordering lemma.

This statement was already made in [12, 16]. However it was interesting to give a somewhat detailed description of the reordering procedure since the explicit computation of most exact eigenstates will necessarily involve reorderings of multispinon states obtained by action of the current algebra operators on the original reordered "base space" vectors. Incidentally (and fortunately since it is a rather cumbersome procedure) this is not required for the lowest lying one- and two-spinon states which are the only ones which we shall consider in the final section.

Remark 1: Further degeneracies occur among the so-called "base states" defined in (42) owing to other quadratic identities such as (41). For instance antisymmetric $R \times R$ doublets $\left(\Phi_{-n}^{\alpha} \Phi_{-n}^{\beta}-\Phi_{-n}^{\beta} \Phi_{-n}^{\alpha}\right)$ vanish. A more exhaustive treatment of the eigenvalue problem requires a consistent definition of an actual basis for the spinon Hilbert space, such as is discussed for instance in [14]. We shall exemplify this construction soon.

Remark 2: One can actually construct the full Hilbert space of the $s u(R)_{1}$ theory by taking only spinon fields in the vector representation $\lambda=\bar{R}$ 14. The KM highest weight representations are then chosen in a consistent way leading to generic states of the form:

$$
\Phi_{-\frac{R-2 q+1}{2 R}-n_{q}}^{\alpha_{q}} \cdots \Phi_{-\frac{R-3}{2 R}-n_{2}}^{\alpha_{2}} \Phi_{-\frac{R-1}{2 R}-n_{1}}^{\alpha_{q}} \mid 0>
$$

where $n_{q} \geq \cdots \geq n_{2} \geq n_{1} \geq 0$. These states are eigenvectors of $L_{0}$ (Virasoro generator) with eigenvalues:

$$
l_{0}=-\frac{q(q-R)}{2 R}+\sum_{i=1}^{q} n_{i}
$$

The other $s u(R)$ spinon representations are obtained from this one as particular concatenations of $l$ antisymmetrized vector operators with the same mode $n_{k}=n_{k+1} \cdots=n_{k+l}$. In this construction the modes $n_{1} \cdots n_{q}$ can always be constrained to obey the ordering condition:

$$
n_{q} \geq n_{q-1} \geq \cdots \geq n_{1}
$$

and $n_{q+R}>n_{q}$ for any value of $q$, otherwise the state is either null or a lower-spinon state. As a consequence the value of the pure-spinon part of the energy-momentum tensor $T_{0}^{s u(R)}(=-($ sum of all modes $))$ has a minimal value for a given $q$, namely when decomposing $q=(R-1) a+b$ one has

$$
T_{0} \geq\left(\frac{R-1}{2 R}\right) a^{2}+\frac{a b}{R}-\frac{b^{2}-b R}{2 R}>\frac{R-1}{2 R} a^{2}
$$


which increases strictly when $q$ increases. As a consequence only a finite number of indepen-

dent multispinon states exist at each level of $T_{0}^{s u(R)}$. This plays a crucial role in our incoming discussion.

This construction now has the advantage of being easier to handle formally since it uses only one type of spinon field with simple fusion rules for $\sigma, \rho$. We shall use it from now on.

\subsection{Action of the Hamiltonian}

We are now in a position to prove that the Hamiltonian (14) can be consistently reduced to finite-dimensional vector spaces and to indicate how to compute its explicit action on such states. The following statements achieve this purpose:

1. The action of $H$ can be reduced consistently to the vector space of states with a fixed negative sum of scalar+spinon modes.

This is an obvious consequence of the fact that, although not a zero-mode of a conformal field, the Hamiltonian is a sum of monomials with overall zero scalar+spinon modes. Hemce the total sum of modes is a good quantum number. It is indeed the eigenvalue of the full energy-momentum tensor $T_{0}$. The reordering lemmas and the Corollary 1 make it sure that a given overall sum may be obtained by only a finite number of combinations of individual modes, hence defines a finite-dimensional subspace of states since we are in the single-spinon representation. This conclusion is also valid for the Yangian generators which also commute with $T_{0}$.

2. The action of $H$ can be further reduced to the vector spaces of states belonging to a given $s u(R)$ representation from the spinon sector.

This is due to the $s u(R)$-scalar nature of the Hamiltonian and enables us to further consistently reduce the dimension of the subspaces of states on which $H$ acts. Note that since $Q_{0}^{a}, Q_{1}^{a}$ are not scalars, this restriction may not apply to computations involving the Yangian.

As illustration we now give an extensive list of the low-lying states which build the small-dimensional Hilbert spaces to be later considered for an explicit diagonalization of the Hamiltonian. We particularize to the case of $S U(3)$ spinons but the generic $S U(R)$ case exhibits the same features. The $S U(3)$ current basis of $\overline{3}$-spinon states used here was constructed in 14.

$$
\begin{aligned}
& T_{0}=0: \quad\left|0>_{\text {spinon }} \otimes\right| 0>_{\text {boson }} \equiv \mid 0>\in \mathbf{1} \\
& T_{0}=\frac{1}{3}: \quad \phi_{-1 / 3}^{\alpha}\left|0>\in \overline{\mathbf{3}} ; \phi_{0}^{\alpha} \phi_{-1 / 3}^{\beta}\right| 0>\in \mathbf{3} \\
& T_{0}=1: \quad \alpha_{-1}\left|0>\in \mathbf{1} ; \quad \phi_{-2 / 3}^{\alpha_{3}} \phi_{0}^{\left[\alpha_{2}\right.} \phi_{-1 / 3}^{\left.\alpha_{1}\right]}\right| 0>\in \mathbf{8} \equiv J_{-1}^{a} \mid 0> \\
& T_{0}=\frac{4}{3}: \quad\left\{\alpha_{-1} \phi_{-1 / 3}^{\alpha_{1}}\left|0>, \phi_{-4 / 3}^{\alpha_{1}}\right| 0>\right\} \in \overline{\mathbf{3}} \\
& \left\{\alpha_{-1} \phi_{0}^{\left[\alpha_{2}\right.} \phi_{-1 / 3}^{\left.\alpha_{1}\right]} \mid 0>\right\} \in \mathbf{3} \\
& \left\{\phi_{0}^{\left(\alpha_{2}\right.} \phi_{-1 / 3}^{\left.\alpha_{1}\right)} \mid 0>\right\} \in \overline{\mathbf{6}} \\
& \left\{\phi_{-1 / 3}^{\left(\left[\alpha_{4}\right.\right.} \phi_{-2 / 3}^{\left.\alpha_{3}\right]} \phi_{0}^{\left[\alpha_{2}\right.} \phi_{-1 / 3}^{\left.\left.\alpha_{1}\right]\right)} \mid 0>\right\} \in \mathbf{6}
\end{aligned}
$$




$$
\begin{aligned}
T_{0}=2: \quad & \left\{\alpha_{-2}\left|0>,\left(\alpha_{-1}\right)^{2}\right| 0>\right\} \in \mathbf{1} \\
& \left\{\alpha_{-1} J_{-1}^{a}\left|0>, \phi_{-5 / 3}^{\alpha_{3}} \phi_{0}^{\left[\alpha_{2}\right.} \phi_{-1 / 3}^{\left.\alpha_{1}\right]}\right| 0>, \phi_{-2 / 3}^{\left[\alpha_{3}\right.} \phi_{-1}^{\left.\alpha_{2}\right]} \phi^{\alpha_{1}}-1 / 3 \mid 0>\right\} \in \mathbf{8} \\
& \left\{\phi_{-2 / 3}^{\left[\alpha_{3}\right.} \phi_{-1}^{\alpha_{2}} \phi^{\left.\alpha_{1}\right]}-1 / 3 \mid 0>\right\} \in \mathbf{1} \equiv J_{-1}^{a} J_{-1}^{a}\left|0>\equiv T_{-2}\right| 0>
\end{aligned}
$$

Not only the Casimirs of the representations themselves but the weights of the particular vectors are conserved, hence one indeed has a consistent reduction to low (2 and 3) dimensional spaces.

3. Only a finite number of monomial operators in $H$ contribute to the action of $H$ for a given mode-number $-N_{0}$.

a) cubic $\alpha$ term: Reordering it as $\alpha_{n_{1}} \alpha_{n_{2}} \alpha_{n_{3}}$ with $n_{1} \geq n_{2} \geq n_{3}$ and $n_{1} \geq 0$, only terms with $n_{1} \leq N_{0}$ may not annihilate states with mode number $N_{0}$. This leaves at most $N_{0}^{2}$ terms acting on this space of states. The same reasoning applies to cubic $J$ terms.

b) $\alpha_{-n} T_{n}^{J}$ terms, $n$ positive or negative: one needs to limit $n$ to be less than $N_{0}$ owing to the action of the $T_{n}^{J}$ on the spinon states and $-n$ also to be less than $N_{0}$ owing to the $\alpha_{-n}$ term to have a non-trivial (i.e. non-cancelling) action. This leaves $2 N_{0}$ terms acting at most.

c) $n \alpha_{-n} \alpha_{n}$ and $n J_{-n}^{a} J_{n}^{a}$ (ordered !) terms with $n>0$. Here again $n$ may not be higher than $N_{0}$ in order to act non-trivially on spinon states by the first terms $\alpha_{n}$ or $J_{n}^{a}$. This leaves at most $N_{0}$ terms acting.

4. The explicit action of any particular monomial on any particular state can be obtained from scalar commutation relations (Wick theorem) and spinon OPE.

The only non-obvious point here is the description of the action of modes of the energy momentum tensor $T_{n}^{J}$ and the current $J_{n}^{a}$ (always involved in scalar combinations) on spinon states. Adjoint action on the spinon modes is a clear consequence of the conformal and $s u(R)$ properties of the spinons:

$$
\begin{gathered}
{\left[T_{n}^{J}, \Phi_{m}^{\Sigma}\right]=(n(\Delta(\lambda)-1)-m) \Phi_{m+n}^{\Sigma}} \\
{\left[J_{n}^{a}, \Phi_{m}^{\Sigma}\right]=t^{a(\lambda)} \cdot \Phi_{n+m}^{\Sigma}}
\end{gathered}
$$

where $t^{a(\lambda)}$ denotes the generators of $s u(R)$ in any appropriate representation for the spinon field $\Phi^{\Sigma}$, in our study specifically the $\bar{R}$ representation. On the other hand the action of $T_{n}^{J}$ and $J_{n}^{a}$ on the spinon vacuum can be obtained in terms of spinon fields through the spinon-spinon OPE. Indeed, together with the constant terms leading to the commutation relation (41) the OPE also contains terms proportional to $J$ and $T^{J}$. This allows to rewrite $J_{n}^{a} \mid 0>_{s p}$ and $T_{n}^{J} \mid 0>_{s p}$ as spinon $R$-linears, as it can be seen on the $S U(3)$ states constructed above. The reordering lemmas are then to be used to get fully reordered states.

As it can be deduced from this description the Hamiltonian may not conserve the overall number of $\overline{3}$ spinons due to the $\alpha T$ coupling and also the arbitrary cubic self-coupling $\gamma= \pm \sqrt{\frac{b^{2}}{R}+1}$ which rescales the $(J)^{3}$ term w.r.t. the $n J^{2}$ term. As emphasized in 14] only for $\gamma=+1$ does the Hamiltonian and the Yangian generators conserve the number of spinons?2 This needs not worry us anyway since our reduction to finite-dimensional spaces

\footnotetext{
${ }^{2}$ We thank the referee for pointing this out to us
} 
of states is not a consequence of a spinon-number conservation.

This ends the general discussions on the spectrum and eigenstates of the Hamiltonian (14). We are now going to give some specific examples of eigenstates and eigenvalues. We shall restrict ourselves to states obtained from covector spinon operators $(\lambda=\bar{R}$ in the notation of (40)). A supplementary relevant commutation relation is here:

$$
\begin{aligned}
{\left[H_{J}, \phi_{-m-\Delta}^{\alpha}\right] } & =\frac{R(R+1)}{2 R-1}\left(1+\gamma \frac{R-2}{R+1}\right)\left(m^{2}+2 \Delta m\right) \phi_{-m-\Delta}^{\alpha} \\
& +\gamma \frac{R C_{3}(R)}{6(R+1)(R+2)} \phi_{-m-\Delta}^{\alpha}
\end{aligned}
$$

which can be called 'one-body' term. Here $H_{J}$ is the pure spin part of $H$ and $C_{3}(R)$ is the third Casimir of the $R$ representation, $C_{3}(R)=-\frac{\left(R^{2}-1\right)\left(R^{2}-4\right)}{R^{2}}$. In addition, we have

$$
\left[H_{J}, \phi_{-p}^{\alpha} \phi_{-q}^{\beta}\right]=(1-\text { body terms })+\sum_{r>0} 2 r\left(t^{a} \phi\right)_{-p-r}^{\alpha}\left(t^{a} \phi\right)_{-q+r}^{\beta}
$$

which can be thought as 'two-body interaction' between spinons. It results into a dynamical $s u(R)$ Calogero-Moser model for the spinons [11, 16]. One gets almost the same result for the complex conjugate representation, $\bar{R}$. The second Casimir is not modified hence $\Delta(\bar{R})=\Delta(R)$. Only the third Casimir changes its sign, $C_{3}(\bar{R})=-C_{3}(R)$.

Once the spectrum is obtained, the particular identification of one amongst the family of Hamiltonians (38) as a collective formulation of the original discrete spin Calogero model may be achieved by looking for a specific behaviour for the eigenvalues. Consistency of the identification in particular requires that they be rational or even integer (up to an overall normalization). This will impose restrictions on the value of $b$ as we shall exemplify in the next section.

\section{Spectrum of the Hamiltonian: one-spinon states}

We finally wish to give a simple example of derivation of eigenvalues and eigenstates as a first application of our construction, reserving a more complete investigation for further studies.

Consider the one spinon sector in the $\bar{R}$ representation. The lowest energy state is given by

$$
\Phi\left(\begin{array}{c}
\bar{R} \\
\bar{R} 0
\end{array}\right)-\Delta\left|0>=\phi_{-\Delta}^{\alpha}\right| 0>
$$

where $\mid 0>$ is the vacuum state satisfying the lowest weight properties:

$$
\phi_{s}^{\alpha}\left|0>=0(s>0) ; J_{n}^{a}\right| 0>=0(n>0) ; T_{m} \mid 0>=0(m \geq-1)
$$

The energy and momentum eigenvalue of (55) is trivially found to be

$$
\begin{aligned}
E_{0} & =\gamma \frac{R C_{3}(\bar{R})}{6(R+1)(R+2)} \\
p_{0} & =\Delta
\end{aligned}
$$


Next consider the lowest excited states with $p=\Delta+1$. The space of these states is spanned by two vectors as seen before on the example of $S U(3)$ :

$$
\begin{aligned}
\alpha_{-1} \phi_{-\Delta}^{\alpha} \mid 0> \\
\phi_{-\Delta-1}^{\alpha} \mid 0>
\end{aligned}
$$

Acting with the Hamiltonian (14) on these states and using notations defined in Section 3 the only nonvanishing terms we find are,

$$
\begin{aligned}
H_{\alpha} \alpha_{-1} \phi_{-\Delta}^{\alpha} \mid 0> & =\alpha_{-1} \phi_{-\Delta}^{\alpha} \mid 0> \\
H_{J} \alpha_{-1} \phi_{-\Delta}^{\alpha} \mid 0> & =\gamma \frac{R C_{3}(\bar{R})}{6(R+1)(R+2)} \alpha_{-1} \phi_{-\Delta}^{\alpha} \mid 0> \\
H_{\text {int }} \alpha_{-1} \phi_{-\Delta}^{\alpha} \mid 0> & =b T_{-1} \phi_{-\Delta}^{\alpha}\left|0>+b \sum_{n>0} \alpha_{-n} T_{n} \alpha_{-1} \phi_{-\Delta}^{\alpha}\right| 0> \\
& =b \phi_{-\Delta-1}^{\alpha} \mid 0> \\
H_{J} \phi_{-\Delta-1}^{\alpha} \mid 0> & =(R+1+\gamma(R-2)) \phi_{-\Delta-1}^{\alpha} \\
& +\gamma \frac{R C_{3}(R)}{6(R+1)(R+2)} \phi_{-\Delta-1}^{\alpha} \mid 0> \\
H_{\text {int }} \phi_{-\Delta-1}^{\alpha} \mid 0> & =b \sum_{n>0} \alpha_{-n} T_{n} \phi_{-\Delta-1}^{\alpha} \mid 0> \\
& =b \sum_{n>0} \alpha_{-n}(n \Delta-n+\Delta+1) \phi_{-\Delta+n-1}^{\alpha} \mid 0> \\
& =b \alpha_{-1}(2 \Delta) \phi_{-\Delta}^{\alpha} \mid 0>
\end{aligned}
$$

where we recall that $H_{\alpha}, H_{J}, H_{\text {int }}$ are pure scalar, pure spin part, and coupling term of $H$, respectively.

Therefore we see that the Hamiltonian restricted to this space is given by

$$
H=\left(\begin{array}{cc}
1+\gamma \frac{R C_{3}(R)}{6(R+1)(R+2)} & 2 \Delta b \\
b & (R+1)+\gamma(R-2)+\gamma \frac{R C_{3}(R)}{6(R+1)(R+2)}
\end{array}\right)
$$

The eigenvalues of this matrix can easily be obtained and are given by

$$
\begin{aligned}
& E_{1}=R+\gamma(R-1)+\gamma \frac{R C_{3}(R)}{6(R+1)(R+2)} \\
& E_{2}=2-\gamma+\gamma \frac{R C_{3}(R)}{6(R+1)(R+2)}
\end{aligned}
$$

What is physically more meaningful is the energy gap:

$$
\begin{aligned}
& E_{1}-E_{0}=R+\gamma(R-1) \\
& E_{2}-E_{0}=2-\gamma
\end{aligned}
$$

We see that this energy gap is rational if and only if $\gamma$ is a rational number.

The more complicated study of two- and multispinon states should later allow a better hold on possible values of $\gamma$. 


\section{Appendices}

\subsection{Appendix A: Normal-ordering and O.P.E.}

We use throughout this paper the normal ordering defined for instance in [17].:

$$
(A 1) \quad(A B)(z)=\frac{1}{2 \pi i} \int \frac{d w}{w-z} A(w) B(z)
$$

where $A$ and $B$ are conformal fields. The most relevant consequence (for our computations) of this definition is the identity connecting the coefficients of the OPE and the normal-ordered commutator:

If the OPE of two conformal fields $A, B$ reads:

$$
A(z) B(w) \equiv \sum_{r>0}^{r_{0}} \frac{\{A B\}_{r}}{(z-w)^{r}}+\text { finite terms }
$$

the normal-ordered commutator reads:

$$
([A, B])(z)=\sum_{r>0}^{r_{0}} \frac{(-1)^{r+1}}{r !} \partial^{r}\{A B\}_{r}(z)
$$

A reordering identity is also very useful in these computations

$$
(A 2) \quad(A(B C))(z)=(B(A C))(z)-(([A, B]) C)(z)
$$

We also recall the relevant OPE used in computing the commutators in Section 3 ( $k$ denotes the central charge of the Kac-Moody algebra; here $k=1$ ):

$$
\begin{aligned}
(O 1): J^{a}(z) J^{b}(w) & =k \frac{\delta_{a b}}{(z-w)^{2}}+f^{a b c} \frac{J^{c}(w)}{z-w}+\cdots \\
(O 2): J^{a}(z) W^{b}(w) & =\frac{2 k+R}{2(z-w)^{2}} d^{a b c} J^{c}(w)+\frac{1}{z-w} f^{a b c} W^{c}(w)+\cdots \\
\text { where } \mathrm{W}^{\mathrm{b}}(\mathrm{w}) & =\frac{1}{2} d^{a b c}\left(J^{a} J^{c}\right)(w) \\
(O 3): J^{a}(z) W(w) & =\frac{k+R}{(z-w)^{2}} W^{a}(w)+\cdots \\
\text { where } \mathrm{W}(\mathrm{w}) & =\frac{1}{6} d^{a b c}\left(J^{a}\left(J^{b} J^{c}\right)\right)(w)
\end{aligned}
$$

\subsection{Appendix B: Normalizations and Jacobi identities for $\operatorname{sl}(R)$}

We introduce a basis for the Lie algebra $\operatorname{sl}(R)$ denoted $\left\{t^{a}, a=1 \cdots R^{2}-1\right\}$. We then define:

$$
d^{a b}=\operatorname{Tr}\left(t^{a} t^{b}\right) ; f^{a b c}=\operatorname{Tr}\left(\left[t^{a}, t^{b}\right] t^{c}\right) ; d^{a b c}=\operatorname{Tr}\left(\left[t^{a}, t^{b}\right]_{+} t^{c}\right)
$$

One usually chooses $\left\{t^{a}, a=1 \cdots R^{2}-1\right\}$ to be an orthonormal basis, hence $d^{a b}=\delta^{a b}$, $f^{a b c}$ is totally antisymmetric and $d^{a b c}$ is totally symmetric.

The most useful identities are then:

a) normalization identities : 


$$
\begin{aligned}
(N 1): d^{a b c} \delta^{a b}=0 & ; \quad(N 2): f^{a b c} f^{d b c}=-2 R \delta^{a d} \\
(N 3): d^{a b c} d^{d b c}=\frac{2 R^{2}-4}{R} \delta^{a d} & ; \quad(N 4): f^{a d b} f^{b e c} f^{c f a}=R f^{d e f} \\
(N 5): \quad d^{a b c} f^{b e c} f^{c f a}=R d^{d e f} & ; \quad(N 6): \quad d^{a d b} d^{b e c} d^{c f a}=\frac{R^{2}-12}{R} d^{d e f}
\end{aligned}
$$

\section{b) Jacobi identities}

$$
\begin{aligned}
& f^{a d e} f^{e b c}+f^{b d e} f^{e c a}+f^{c d e} f^{e a b}=0 \\
& f^{a d e} d^{e b c}+f^{b d e} d^{e c a}+f^{c d e} d^{e a b}=0 \\
& f^{a b c} f^{c d e}-d^{b d c} d^{c a e}+d^{a d c} d^{c d e}=\frac{4}{R}\left\{\delta^{a e} \delta^{b d}-\delta^{a d} \delta^{b e}\right\}
\end{aligned}
$$

\subsection{Appendix C: Null fields}

It was established in 15 that the following two fields are in fact null conformal fields and this property is extensively used in showing the cancellation of the relevant commutators in Section 3:

$$
\begin{aligned}
(F 1): \Xi^{a}(w) & =\frac{1}{6} f^{a b c} f^{c d e}\left(\partial J^{b}\left(J^{d} J^{e}\right)-J^{b}\left(\partial J^{d} J^{e}\right)\right)(w) \\
& +\frac{R^{2}}{4(R+2)^{2}} d^{a b c}\left(-\frac{2}{3}\left(W^{b} \partial J^{c}+\partial J^{b} W^{c}\right)+\frac{1}{3}\left(\partial W^{b} J^{c}+J^{b} \partial W^{c}\right)\right) \\
& +\frac{1}{24} R(R+2) \partial^{3} J^{a}(w) \\
(F 2): \Phi^{a d}(w) & =\left(f^{a h p} d^{p b d}-f^{d h p} d^{p b a}\right)\left(J^{b} W^{h}\right)(z) \\
& +(R+2)\left(\partial J^{d} J^{a}-\partial J^{a} J^{d}\right)(z)
\end{aligned}
$$

\subsection{Appendix D: Relevant commutators}

We make an extensive use of the pure-spin results in [15]. The relevant commutators, easily computed or derived from these results, are:

$$
\begin{aligned}
(C 1):\left[f^{a b c} \sum_{m>0} J_{-m}^{b} J_{m}^{c}, \sum_{m>0} m J_{m}^{a} J_{-m}^{a}\right] & =\frac{1}{3} f^{a b c} f^{c d e}\left(\partial J^{b}\left(J^{d} J^{e}\right)-J^{b}\left(\partial J^{d} J^{e}\right)\right)_{0} \\
(C 2):\left[f^{a b c} \sum_{m>0} J_{-m}^{b} J_{m}^{c}, \frac{R}{(R+1)(R+2)} W_{0}\right] & -\frac{R}{R+2}\left[W_{0}^{a}, \sum_{m>0} m J_{m}^{a} J_{-m}^{a}\right]=0
\end{aligned}
$$

This is obtained by immediate computation using the commutators derived from the OPE $O 2$ and $O 3$ in Appendix 1. 


$$
\begin{aligned}
(C 3): \quad & -\frac{R^{2}}{(R+1)(R+2)^{2}}\left[W_{0}^{a}, W_{0}\right]=\frac{R(R+2)}{12} \partial^{3} J_{0}^{a} \\
& +\frac{R^{2}}{2(R+2)^{2}} d^{a b c}\left(\left(-\frac{2}{3}\left(W^{b} \partial J^{c}+\partial J^{b} W^{c}\right)+\frac{1}{3}\left(\partial W^{b} J^{c}+J^{b} \partial W^{c}\right)\right)\right)
\end{aligned}
$$

This commutator is obtained from a direct evaluation of the l.h.s. using the definition of $W^{a}$ as $\frac{1}{2} d^{a b c}\left(J^{b} J^{c}\right)$, the definition $(A 1)$ of normal-ordering and the commutator O3. One then gets:

$$
\text { (l.h.s.) }=\frac{\mathrm{R}^{2}}{(\mathrm{R}+2)^{2}} \mathrm{~d}^{\mathrm{abc}}\left(\sum_{\mathrm{n}>0} \mathrm{nW}_{-\mathrm{n}}^{\mathrm{b}} \mathrm{J}_{\mathrm{n}}^{\mathrm{c}}-\mathrm{nJ}_{-\mathrm{n}}^{\mathrm{b}} \mathrm{W}_{\mathrm{n}}^{\mathrm{c}}\right)
$$

One then computes the normal-ordered term $d^{a b c} \cdots$ in the r.h.s., using again the normalordering definition $A 1$ :

$$
\begin{aligned}
(\text { r.h.s. }) & =\frac{R^{2}}{2(R+2)^{2}} d^{a b c}\left(\sum_{n>0} 2 n W_{-n}^{b} J_{n}^{c}-2 n J_{-n}^{b} W_{n}^{c}\right) \\
& -\frac{R^{2}}{(R+2)^{2}} d^{a b c}\left[W_{-1}^{b}, J_{1}^{c}\right] \text { (residual term from normal ordering) } \\
& +\frac{R^{2}}{(R+2)^{2}} \partial^{3} J_{0}^{a}
\end{aligned}
$$

and the last two terms cancel using $O 2$ in Appendix A.

Finally Serre's relations for the Yangian generators use the following commutators computed in 15]:

$$
\begin{aligned}
(C 4):\left[f^{a b c} \sum_{m>0} J_{-m}^{b} J_{m}^{c}, f^{d e f} \sum_{n>0} J_{-m}^{e} J_{m}^{f}\right] & =\frac{1}{6}\left(f^{a b c} f^{c e g} f^{d e f}-(a \leftrightarrow d)\right)\left(\left(J^{b}\left(J^{f} J^{g}\right)\right)_{0}-\left(J_{0}^{g} J_{0}^{f} J_{0}^{b}\right)\right) \\
(C 5):\left[f^{a b c} \sum_{m>0} J_{-m}^{b} J_{m}^{c}, W_{0}^{d}\right] & -(a \leftrightarrow d)=0 \\
(C 6):\left[W_{0}^{a}, W_{0}^{d}\right] & =\left(d^{a b c} d^{\text {def }} f^{c e g}-d^{d b c} d^{a e f} d^{c e g}\right)\left(J^{b}\left(J^{f} J^{g}+J^{g} J^{f}\right)\right)_{0} \\
& +(R+2) d^{a b c} d^{d e f}\left(\partial J^{b} J^{f}-\partial J^{f} J^{b}\right)_{0}
\end{aligned}
$$

\section{Acknowledgements}

This work was sponsored by CNRS; CNRS-NSF Exchange Programme AI 06-93; US DOE Contract DE-FG02-91ER40688 Task A. J.A. wishes to thank Brown University Physics Department for their hospitality. A.J. thanks LPTHE Paris VI/VII for their hospitality.

\section{References}

[1] J. Avan, A. Jevicki; Nucl. Phys. B 469 (1996), 287. 
[2] F.D.M. Haldane, Phys. Rev. Lett. 60 (1988), 635; B.S. Shastry, ibid. p. 639; V.I. Inozemtsev, J. Stat. Phys 59, 1143 (1990).

[3] F. Calogero, Lett. Nuov. Cim. 13 (1975), 411; J. Moser, Adv. Math. 16 (1976), 1; B. Sutherland, Phys. Rev. Lett. 34 (1975), 1083.

[4] J. Gibbons, T. Hermsen, Physica D 11 (1984), 337; S. Wojciechowski, Phys. Lett. A 111 (1985), 101.

[5] A. P. Polychronakos, Phys. Rev. Lett 69 (1992), 703.

[6] Z.N.C. Ha, F.D.M. Haldane; Phys. Rev. B 46 (1992), 9359.

[7] D. Bernard, K. Hikami, M. Wadati; Proceedings of the VIth Nankai Workshop (1994).

[8] D. Bernard, M. Gaudin, F.D.M. Haldane, V. Pasquier, J. Phys. A (Math. Gen) 26 (1993), 5219.

[9] E.Billey, J. Avan, O. Babelon; Phys. Lett A 188 (1994), 263; J. Avan, O. Babelon, E. Billey, Comm. Math. Phys, 178 (1996), 281.

[10] F.D.M. Haldane, Z.N.C. Ha, J.C. Talstra, D. Bernard, V. Pasquier, Phys. Rev. Lett. 69 (1992), 2021.

[11] D. Bernard, V. Pasquier, D. Serban, Nucl. Phys. B 428 (1994), 612.

[12] P. Bouwknegt, A.W.W. Ludwig, K. Schoutens, Phys. Lett. B 338 (1994), 448.

[13] F.D.M. Haldane, Phys. Rev. Lett. 67 (1991), 937.

[14] P. Bouwknegt, K. Schoutens, hep-th 9607064.

[15] K. Schoutens, Phys. Lett. B 331 (1994),335.

[16] C.H. Ahn, S.K. Nam, hep-th 9510242.

[17] F.A. Bais, P. Bouwknegt, M. Surridge, K. Schoutens, Nucl. Phys. B 304 (1988), 348.

[18] B. Sakita, hep-th 9607047.

[19] V.G. Drinfel'd, Proceedings of the I.C.M. 1986, Berkeley, pp. 798-820.

[20] A. Jevicki, B. Sakita, Nucl.Phys. B 165 (1980), 511; S.R. Das, A. Jevicki, Mod. Phys. Lett. A 5 (1990), 1639.

[21] J. Polchinski, Nucl. Phys. B 346 (1990), 253; ibid. B 362 (1991), 25.

[22] J. Avan, E. Billey, Phys. Lett. A 198 (1995), 183.

[23] N. Yu. Reshetikhin, J. Phys. A 24 (1991), 3299.

[24] D. Cabra, G. Rossini, hep-th 9602041. 